Ingeniería Investigación y Tecnología, ISSN 2594-0732 V. 4. 299-309, 2004

(artículo arbitrado)

DOI:http://dx.doi.org/10.22201/fi.25940732e.2004.05n4.020

\title{
Dirigibles: aerostática y problemas de cálculo
}

\author{
R. Ávila-Rodríguez y J. Cervantes de Gortari \\ Facultad de Ingeniería, U N A M \\ E-mail: ravila@servidor.unam.mx
}

(recibido: marzo de 2002; aceptado: septiembre de 2003)

\begin{abstract}
Resumen
Se presentan las bases termodin ámicas que permiten llevar a cabo el an áisis aerostático de un diri gible. Se fundamenta y se extiende la teoría simplificada que fue incorporada en el desarrollo de una regla de cálculo utilizada en la navegación de diri gi bles a partir de 1923. Se resuelven tres de los problemas que fueron propuestos por los diseñadores de la regla de cálculo para verificar su utilidad. La solucó $n$ de los ejemplos a partir del uso de la teońa formulada en este trabajo se compara con los resultados obtenidos con la regla de cá lculo.
\end{abstract}

Descriptores: diri gi bles, aerostática, termodinámica, blimps, flotación, atmósfera estándar.

\begin{abstract}
The ther mo dy nam ics theory that al lows to per form theaerostaticanal $y$ sis of an air ships is pre sented. The sim ple the ory that was in volved in the de sign of an air ship slide rule, which was used in theair ship navi ga tion from 1923, is founded and ex tended. Threeof theprob lems that were pro posed by theslideruledesign ers, to ver ify its use ful ness, aresolved. Thesolution of the prob lems using thetheory presented in this pa per, is com pared with theresults ob tained by the slide rule.
\end{abstract}

Key words: air ships, aerostatic, ther mo dy namics, blimps, lift, stan dard atmosphere.

\section{Introducción}

La tecnología de los di ri gi bles tuvo su origen a finales del siglo XIX y alcanzó su etapa de desarrollo durante las primeras tres décadas del siglo veinte. El dramático incidente del dirigible alemán Hindenburg ocurrido en Lakehurst, Nueva Jersey, EUA, el 6 de mayo de 1937, marcó el fin de la primera era de los gigantes voladores (White, 1976), (Meyer, 1980) y (Kleinheins, 1985).

El desarrollo que han alcanzado durante los últimos años la ciencia y la tecnología de los materiales y los sistemas computacionales de predicción de las características de la atmósfera, permite ofrecer solución a muchos de los problemas a los que se enfrentaban los investigadores e ingenieros que hicieron posible el vuelo de los primeros dirigibles.

Los dirigibles actuales y del futuro no deben ser considerados únicamente como competidores de los medios convencionales de transporte, sino que al tomar en cuenta sus características (seguridad, bajo 
costo de operación, estabilidad y gran independencia de vuelo), deben ser identificados como naves que pueden ser utilizadas en diversas aplicaciones. Una nueva era de los diri gibles dará inicio en el momento en que los usuarios potenciales de estas naves, tomen conciencia de las múltiples ventajas que ofrecen en cualquiera de los siguientes campos (Rodríguez y Rodríguez, 1987), (Jacobo y Zimán, 1984), (Sahlberg, 1986), (Hillsdon, 1992), (Taylor, 1992), (Hayes, 1994), (Delahunt, 1997):

1 Observación: Fotografía aérea, vigilancia policial, servicio de guardacostas, detección de incendios forestales, monitoreo de tráfico de vehículos, medición de contaminantes en la atmósfera, inspección de ductos petroleros, detección de yacimientos minerales, inspección de las actividades de pesca y caza.

2 Comunicación: Plataformas aéreas para la transmisión de estaciones de radio y TV.

3 Agricultura: Dispersión de fertilizantes y semillas, fumigación de bosques y terrenos de siembra, ayuda a combatir incendios forestales.

4 Transporte: Mercancías, alimentos y maquinaria hacia zonas de desastre 0 de difícil acceso y rescate de damnificados.

5 Grúa móvil: Levantamiento de postes, tuberías, maquinaria, mate rial de construcción y elevación de materiales de gran peso a grandes alturas.

6 Investigación científica: Meteorología, geofísica, oceanografía e investigación de la vida salvaje animal.

7 Aplicaciones militares: Aviso aéreo oportuno mediante antenas de radar, defensa antisubmarina y búsqueda marítima de minas.
El presente trabajo tiene como propósito principal, formular las bases te óricas (aerostática y termodinámica), que permiten llevar a cabo el análisis aerostático de un dirigible. A partir de la teoría mostrada y de las ecuaciones resultantes, el estudiante de ingeniería o de física podrá iniciar, al menos desde el punto de vista aerostático, el estudio del comportamiento de cuerposdirigibles.

La historia reciente de los dirigibles no se presenta en este artículo, ya que existen una gran variedad de publicaciones al respecto. En la revista AIRSHIPS (The Jour nal of the Air ships Association) se ha reportado lo más relevante de los últimos años. Es conveniente mencionar que en México, dos empresas impulsaron de manera decidida el desarrollo de di rigi bles. Una de ellas, la empresa Estrategia, S.C. de los hermanos Rodríguez, llevó a cabo un interesante estudio relacionado con la factibilidad de conformar una flota de dirigibles en nuestro país (Rodríguez y Rodríguez, 1987). La empresa Spacial, fundada por Mario Sánchez, construyó en la década de los 80's tres prototipos en forma lenticular (MLA-24A, MLA-32A y MLA) (Lana, 1991). Lamentablemente Mario Sánchez falleció en un accidente automovilístico en 1990, por lo que la construcción del dirigible lenticular quedó sin concluir y el desarrollo de los dirigibles en México, durante la década de los 90's, quedó en el olvido.

En la segunda sección, se presenta la teoría termodinámica que permite llevar a cabo el análisis aerostático de un dirigible que se encuentra en condiciones tensas (volumen de la nave totalmente lleno) y en condiciones flexibles (volumen parcialmente lleno). Se presenta la estabilidad aerostática del di rigible cuando pierde o gana lastre, y cuando la temperatura del gas y del aire atmosférico cambian de manera repentina. En la tercera 
sección, se presenta la solución de tres de los problemas que fueron formulados por los diseñadores de una regla de cálculo, la cual fue utilizada por los pilotos de los dirigibles a partir de 1923 (Weaver y Pickering, 1923). La solución obtenida a partir de la teoría formulada en el presente estudio se compara con la solución obtenida con el uso de la regla de cálculo.

\section{Aerostática de un dirigible}

Debido a que los aeroplanos convencionales no están sujetos a fuerzas de flotación, la aerostática no se considera como una rama de la teoría aerodinámica. Para comprender la aerodinámica de los dirigibles es importante conocer, en primera instancia, sus características aerostáticas (Arnstein y Klemperer, 1936).

La fuerza de flotación que produce el gas (hidrógeno o helio) que se encuentra dentro del di rigible, siempreactúa en la dirección verti calz. El equilibrio ver ti cal entre el peso del dirigible y la capacidad de flotación puede alcanzarse de manera independiente al equilibrio de las fuerzas (empuje y arrastre) presentes en la dirección axial. El estado de equilibrio aerostático de un dirigible depende, tanto del gradiente de temperaturas de la atmósfera $\mathrm{dT}$ aire $/ \mathrm{dz}$, como del volumen ocupado por el gas (dirigible totalmente lleno "taut" 0 dirigible parcialmente lleno "imp") (Prandtl y Tietjens, 1957).

Antiguamente, los pilotos de los dirigibles hacían uso de reglas de cálculo para determinar el equilibrio aerostático de la nave (Arnstein y Klemperer, 1936), (Weaver y Pickering, 1923).

En el presente trabajo se presenta la teoría termodinámica que permite llevar a cabo el análisis aerostático de un dirigible. Las ecuaciones que se presentan se utilizan para resolver tres de la serie de problemas que fueron propuestos por Weaver y Pickering (1923) para que los pilotos de los Blimps norteamericanos, de principios de la década de los 20's, aprendieran el uso de la regla de cálculo.

En la solución de los problemas propuestos, se supone que el di ri gi ble se desplaza en una atmósfera estándar.

\section{Atmósfera estándar}

La atmósfera estándar es una distribución vertical hipotética de la presión, temperatura y densidad de la atmósfera. A través de un acuerdo internacional, este modelo se toma como representativo de la atmósfera para propósitos de calibración de altímetros, para el cálculo del comportamiento de aviones, diseño de misiles, etc. En este modelo de atmósfera se supone que el aire se comporta como un gas ideal que satisface la ecuación de la hidrostática. Las ecuaciones que relacionan la variación (con respecto a la altura) de los campos de presión, temperatura y densidad del aire atmosférico son (a) la ecuación de estado de un gas ideal

$$
\frac{P}{\rho_{\text {aire }}}=R_{\text {aie }} T_{\text {aire }}
$$

y (b) la ecuación de la hidrostática

$$
\frac{d P}{d z}=-\rho_{\text {aire }} g
$$

donde $P$ es la presión $\left[\mathrm{N} / \mathrm{m}^{2}\right]$, $\rho_{\text {aire }}$ es la densidad $\left[\mathrm{kg} / \mathrm{m}^{3}\right]$, $T$ [K] es la temperatura, $g$ $\left[\mathrm{m} / \mathrm{s}^{2}\right]$ es la aceleración de la gravedad y $R_{\text {aire }}$ $[\mathrm{J} / \mathrm{kg}-\mathrm{K}]$ es la constante del aire evaluada a partir de $R_{\text {aire }}=R / M_{\text {aire }}$ donde $R$ es la constante 
uni ver sal de los gases $(8.31451 \mathrm{~kJ} /(\mathrm{kgmole} \cdot \mathrm{K}))$ $\mathrm{y} \mathrm{M}_{\text {aire }}$ es la masa molar media del aire $(28.965$ $\mathrm{kg} / \mathrm{kgmole})$.

En el modelo de atmósfera estándar, se supone además que el aire no contiene vapor de agua y que la aceleración de la gravedad $g$ no cambia con respecto a la altura. En la definición de la atmósfera estándar se consideran diversos parámetros físicos; sin embargo, para el estudio de la flotación de dirigibles (y para la solución de los problemas propuestos por Weaver y Pickering (1923)), los valores más relevantes son los siguientes:

1 Constante del aire (sin humedad) $R_{\text {aire }}=287.04 \mathrm{~J} / \mathrm{kg}-\mathrm{K}$

2 Aceleración de la gravedad $g=9.80665$ $\mathrm{m} / \mathrm{s}^{2}$

3 Variación de la temperatura con respecto a la altura (Environmental Lapse Rate), $-\mathrm{dT} / \mathrm{dz}=0.0065 \mathrm{~K} / \mathrm{m}$.

Si el aire de la atmósfera se supone que es una sustancia compresible simple (el único modo de trabajo reversible importante es el de compresión), su estado termodinámico queda completamente determinado a partir de la especificación de dos propiedades termodinámicas independientes (Reynolds y Perkins, 1977).

En el presente estudio, se supone que el comportamiento termodinámico de la atmósfera se representa por un proceso politrópico (en donde existe equilibrio hidrostático y se presenta un gradiente de temperaturas $\mathrm{dT} / \mathrm{dz}$ constante) definido por

$$
P\left(\frac{1}{\rho_{\text {aire }} g}\right)^{n}=\text { cte }
$$

donde $\mathrm{n}$ es el exponente politrópico, por lo que al utilizar la ecuación del gas ideal (1) y la ecuación de la hidrostática (2) se obtiene la variación de la presión, temperatura y densidad del aire como función de la altura $z$ (Prandtl y Tietjens, 1957):

$$
\begin{aligned}
& P=P_{1}\left[1+\frac{d T}{d z} \frac{z}{T_{\text {aire }}}\right]^{\frac{n}{n-1}} \\
& T_{\text {are }}=T_{\text {are }}+\frac{d T}{d z} z
\end{aligned}
$$

$$
\rho_{\text {are }}=\rho_{\text {aire }}\left[1+\frac{d T}{d z} \frac{z}{T_{\text {dire }_{1}}}\right]^{\frac{1}{n-1}}
$$

donde $P_{1}, T_{\text {are }_{1}}$ y $\rho_{\text {aira }}$ corresponden a una altura de referencia $z_{1} y \mathrm{dT} / \mathrm{dz}$ se de fine como

$$
\frac{d T}{d z}=-\left(\frac{n-1}{n}\right) \frac{g}{R_{\text {aire }}}
$$

A partir de la ecuación (7), se obtiene que el coeficiente politrópico de una atmósfera estándar es $n=1.234$. Si la atmósfera se rige por un proceso adiabático reversible (isentrópico) y se supone que sus calores específicos son constantes, el coeficiente politrópico es $n=$ $k_{\text {aire }}=1.4$. Utilizando la ecuación (7), se obtiene que el gradiente de temperaturas en la dirección ver ti cal en una atmósfera adiabática sin humedad es $-\mathrm{dT} / \mathrm{dz}=0.00976 \mathrm{~K} / \mathrm{m}$.

Las fuerzas presentes en el estudio del equilibrio aerostático de un di ri gi ble son la fuerza de flotación, debido al volumen desplazado de aire $F_{B}$, el peso total del dirigible $F_{T}$ (tripulación, lastre, motores, combustible, envolvente, etc.) y 
la fuerza resultante $F_{R}$ que motiva el ascenso 0 descenso de la nave, es decir:

$$
F_{R}=F_{B}-F_{T}
$$

donde la fuerza de flotación del dirigible se expresa como

$$
F_{B}=\left(\gamma_{\text {aire }}-\gamma_{\text {gas }}\right) \text { Vol }
$$

donde $V$ ol es el volumen del dirigible.

En este estudio se supone que también el gas se comporta como un gas ideal, por lo que su constante se define como

$$
R_{\text {gas }}=\frac{R}{M_{\text {gas }}} \equiv J / k g-K
$$

donde $M_{\text {gas }}=2.016 \mathrm{~kg} / \mathrm{kgmole}$ y $M_{\text {gas }}=4.003$ $\mathrm{kg} / \mathrm{kgmole}$ es la masa molar media del hidrógeno y del helio respectivamente.

Los pesos específicos del aire y del gas que aparecen en la ecuación (9) se definen como:

$$
\begin{aligned}
& \gamma_{\text {a ire }}=\rho_{\text {aire }} g \\
& \gamma_{g a s}=\rho_{g \text { as }} g
\end{aligned}
$$

Dos casos son analizados en el presente trabajo, uno de ellos es cuando el dirigible se encuentra en condición tensa "taut" (dirigible totalmente lleno de gas) y la otra es cuando se encuentra en condición flexible "limp" (dirigible parcialmente lleno de gas) esta última condición es la que les dió el nombre de Blimps a los dirigibles flexibles de la marina norteamericana y de la Gran Bretaña (Avila y Martínez, 2001). En el estudio de ambos casos se supone que la presión del gas dentro del di ri gible es igual a la presión local del aire atmosférico (Ecuación 4) y que el gas dentro del di ri gi ble se expande siguiendo un proceso adiab ático reversible(isentrópico), por lo que se satisface la siguiente expresión

$$
p\left(\frac{1}{\gamma_{g a s}}\right)^{k}=\text { cte }
$$

donde k es el coeficiente politró pico del gas, correspondiente a un proceso isentrópico.

\section{Aerostática de un dirigible en estado tenso}

En esta condición de operación de la nave, se supone que el dirigible se encuentra totalmente lleno de gas y que el volumen que ocupa el gas es constante. La estabilidad hidrostática del dirigible se obtiene al derivar con respecto a la altura la ecuación (8), con la condición de que el volumen permanece constante (Prandtl y Tietjens, 1957),

$$
\frac{\mathrm{d} F_{R}}{\mathrm{dz}}=\frac{\mathrm{d} F_{B}}{\mathrm{dz}}-\frac{\mathrm{d} F_{T}}{\mathrm{dz}}
$$

En el análisis se supone que el peso total del dirigible $\left(F_{T}\right)$, no varía con respecto a la altura, por lo que su derivada es cero. Por tanto, se tiene lo siguiente

$$
\frac{\mathrm{d} F_{R}}{\mathrm{dz}}=\mathrm{V} \text { ol } \frac{\mathrm{d}}{\mathrm{dz}}\left(\gamma_{\text {aire }}-\gamma_{\text {gas }}\right)
$$

la cual puede escribirse como (haciendo uso de la ecuación de la hidrostática (Ecuación 2)):

$$
\begin{gathered}
\frac{\mathrm{d} F_{R}}{\mathrm{dz}}=\mathrm{V} \text { ol } \frac{\mathrm{dP}}{\mathrm{dz}} \frac{\mathrm{d}}{\mathrm{dP}}\left(\gamma_{\text {aire }}-\gamma_{\text {gas }}\right)= \\
-\mathrm{V} \text { ol } \gamma_{\text {aire }} \frac{\mathrm{d}}{\mathrm{dP}}\left(\gamma_{\text {ai re }}-\gamma_{\text {gas }}\right)
\end{gathered}
$$

Utilizando las ecuaciones (3) y (13) se puede escribir 


$$
\begin{aligned}
\frac{d \gamma_{\text {aire }}}{d P} & =\frac{1}{n} \frac{\gamma_{\text {aie }}}{P} \\
\frac{d \gamma_{\text {gas }}}{d P} & =\frac{1}{k} \frac{\gamma_{\text {gas }}}{P}
\end{aligned}
$$

Sustituyendo las ecuaciones (17) y (18) en la ecuación (16), se tiene (Prandtl y Tietjens, 1957),

$$
\frac{d F_{R}}{\mathrm{dz}}=-\mathrm{V} \text { ol } \frac{\gamma_{\text {aire }}}{\mathrm{P}}\left(\frac{\gamma_{\text {aire }}}{\mathrm{n}}-\frac{\gamma_{\text {gas }}}{\mathrm{k}}\right)
$$

Al integrar la ecuación (19), será posible conocer la fuerza resultante que actúa sobre el dirigiblecuando el piloto asciende 0 desciende la nave. La integral de la ecuación (19) puede escribirse como (Prandtl y Tietjens, 1957):

$$
F_{R}-F_{R 1}=\operatorname{Vol}\left[\gamma_{\text {aire }_{2}}-\gamma_{\mathrm{gas}_{2}}\right]-F_{B_{1}}
$$

donde el primer término del lado derecho es la fuerza de flotación en la nueva posición verti cal, es decir:

$$
F_{B_{2}}=\mathrm{Vol}\left[\gamma_{\text {aire }_{2}}-\gamma_{\mathrm{gas}_{2}}\right]
$$

mientras que la fuerza resultante $F_{R_{1}}$ en la posición de referencia $z_{1}$ se escribe como (Ecuación 8)

$$
F_{R_{1}}=F_{B_{1}}-F_{T_{1}}
$$

El cambio de la fuerza de flotación $\Delta F_{B_{1}}$, se evalúa al derivar la ecuación (9) con respecto a la variación de la temperatura del gas y del aire. Si se supone que la variación de la

$$
\Delta F_{B_{1}}=\frac{\partial F_{B 1}}{\partial T_{\text {gas }_{1}}} \Delta T_{\text {gas } 1_{1}}+\frac{\partial F_{B 1}}{\partial T_{\text {aire } 1}} \Delta T_{\text {aire }}=
$$

temperatura en $z_{1}$ de ambos fluidos es pequeña $\left(\Delta T_{\text {arre }_{1}}\right.$ y $\left.\Delta T_{\text {ga }_{1}} \rightarrow 0\right)$, puede escribirse (Prandtl y Tietjens, 1957),

$$
\mathrm{V} \text { ol }\left[\frac{\gamma_{g_{a_{1}}}}{T_{\text {gas }}} \Delta T_{g a ~ s 1}-\frac{\gamma_{\text {aire }_{1}}}{T_{\text {a ire }_{1}}} \Delta \mathrm{T}_{\text {aire1 }}\right]
$$

La variación de la densidad del gas como función de la altura se determina a partir de

$$
\rho_{\text {gas }_{2}}=\rho_{g_{\text {aq }}}(1-a \Delta z)^{\frac{n}{k(n-1)}}
$$

donde la vari able a se de fine como

$$
a=\left(\frac{n-1}{n}\right) \frac{g}{R_{\text {aire } \text { a ire }_{1}}}
$$

\section{Aerostática de un dirigible e n estado flexible}

En esta condición, el gas dentro del dirigible ocupa un volumen variable. En el análisis se supone que la masa del gas se mantiene constante, es decir, no se libera gas hacia la atmósfera cuando la nave se desplaza en la dirección vertical.

El peso del gas dentro del di ri gi blese de fine como

$$
Q_{\text {gas }}=m_{g a s} g=\rho_{g s} g \vee O_{g a s}=\gamma_{g a ~ s} V_{g a s}
$$

donde $m_{\text {gas }} \mathrm{y} \mathrm{V} \mathrm{ol}_{\text {gas }}$ son la masa y el volumen del gas respectivamente. Utilizando la ecuación (26), la expresión (8) puede escribirse como

$$
F_{R}=Q_{\text {gas }}\left(\frac{\gamma_{\text {aire }}}{\gamma_{\text {gas }}}-1\right)-F_{T}
$$


La estabilidad hidrostática de un di ri gi ble en estado flexible, se obtiene al derivar la ecuación (27) con respecto a la dirección vertical, manteniendo $Q_{\text {gas }}$ como constante, es decir,

(28)

$$
\frac{\mathrm{d} F_{R}}{\mathrm{dz}}=Q_{\text {gas }} \frac{\mathrm{d}}{\mathrm{dz}}\left(\frac{\gamma_{\text {aire }}}{\gamma_{\text {gas }}}-1\right)
$$

Siguiendo el mismo desarrollo a partir del cual se obtuvo la ecuación (19), se llega a la siguiente expresión (Prandtl y Tietjens, 1957),

(29)

$$
\frac{\mathrm{d} F_{R}}{\mathrm{dz}}=-\frac{Q_{\mathrm{gas}} \gamma^{2} \text { aire }}{\gamma_{\mathrm{gax}} \mathrm{P}}\left(\frac{1}{\mathrm{n}}-\frac{1}{\mathrm{k}}\right)
$$

cuya in te gral es

$$
F_{R}-F_{R_{1}}=Q_{\mathrm{gas}}\left[\frac{\gamma_{\text {arre }_{1}}}{\gamma_{\text {gas }_{1}}}(1-\mathrm{a} \Delta \mathrm{z})^{\frac{k-n}{k(n-1)}}-1\right]-F_{B_{1}}
$$

donde (Ecuación 27)

$F_{B_{1}}=Q_{\mathrm{gas}}\left(\frac{\gamma_{\text {aire }_{1}}}{\gamma_{\text {gass }}}-1\right)=\mathrm{V} \mathrm{ol}_{\text {gas }_{1}}\left(\gamma_{\text {aire }_{1}}-\gamma_{\text {gas }}\right)$

es la fuerza de flotación en la posición de referencia $z_{1}$. Utilizando la relación de pesos específicos del aire y del gas, puede escribirse

(32)

$$
(1-\mathrm{a} \Delta \mathrm{z})^{\frac{\mathrm{k} n}{\mathrm{k(n-1)}}}=\left(\frac{\gamma_{\mathrm{aire}_{2}}}{\gamma_{\text {aire }}} \cdot \frac{\gamma_{\mathrm{gas}_{1}}}{\gamma_{\mathrm{gas}_{2}}}\right)
$$

Utilizando la ecuación (32), en la expresión (30), se llega a lo siguiente

$$
F_{R}-F_{R}=F_{B_{2}}-F_{B_{1}}
$$

donde

$F_{B 2}=Q_{g \rtimes}\left(\frac{\gamma_{\text {aire }_{2}}}{\gamma_{\text {gas }_{2}}}-1\right)=\mathrm{Vol}_{\mathrm{gas} 2_{2}}\left(\gamma_{\text {aire } 2}-\gamma_{\mathrm{gas2}_{2}}\right)$

es la fuerza de flotación en la nueva posición vertical.

Si en la posición de referencia $z_{1}$, el piloto decide aumentar 0 disminuir el peso del dirigible en una cantidad $\Delta \mathrm{F}_{\mathrm{T}_{1}}$ y la fuerza de flotación cambia en una cantidad $\Delta \mathrm{F}_{B_{1}}$, la distancia que se desplaza la nave, para lograr el equilibrio hidrostático $\mathrm{F}_{\mathrm{R}}=0$ en la nueva posición, se obtiene a partir de la expresión (30), la cual puede escribirse como

$$
\begin{gathered}
Q_{\text {gas }}\left[\frac{\gamma_{\text {aire1 }}}{\gamma_{\text {gg }_{1}}}(1-\mathrm{a} \Delta \mathrm{z})^{\frac{k-n}{k(n-1)}}-1\right]= \\
F_{T_{1}}+\Delta F_{T_{1}}-\Delta F_{B_{1}}
\end{gathered}
$$

La ecuación (35) tiene la siguiente solución para $\Delta z$

$\Delta \mathrm{Z}=\frac{1}{\mathrm{a}}\left[1-\left[\frac{\gamma_{\mathrm{gas}_{1}}}{\gamma_{\text {are }}}\left(\frac{F_{T_{1}}+\Delta F_{T_{1}}-\Delta F_{B 1}}{Q_{9 \mathrm{~s}}}+1\right)\right]^{\frac{\mathrm{kn}-1)}{\mathrm{k}-\mathrm{n}}}\right](3$

6)

La variación de la fuerza de flotaci ón $\Delta \mathrm{F}_{\mathrm{B}_{1}}$ en las ecuaciones (35) y (36) se obtiene (en el estado "limp") a partir de la ecuación (23), y se escribe como: 
(37)

$$
\Delta F_{B_{1}}=Q_{\text {ga }}\left[\frac{\Delta T_{\text {gas }}}{\sigma T_{\text {aira }}}-\frac{T_{\text {gaf }_{1}} \Delta T_{\text {aire }_{1}}}{\sigma T_{\text {aire }_{1}}^{2}}\right]
$$

donde $\sigma=R_{\text {aire }} / R_{\text {gas }}$

El volumen del gas en la posición vertical $\Delta z$ se obtiene a partir de la relación (26), es decir,

$$
\mathrm{Vol}_{\mathrm{gas} 2}=\frac{\mathrm{m}_{\mathrm{gax}}}{\rho_{\text {ga }}}
$$

donde $\rho_{\text {gas }}$ se obtiene a partir del la ecuación (24).

\section{Solución de problemas propuestos}

Weaver y Pickering (1923), propusieron una serie de problemas que tenían que re solver los pilotos de los Blimps norteamericanos para que aprendieran a utilizar una regla de cálculo, la cual senvía para determinar la aerostática del di ri gi ble. El desarrollo de la regla fue solicitado por la marina de los EUA, debido al éxito que tuvo la regla Scott-Teed, desarrollada y utilizada por los pilotos de los dirigibles Británicos (Weaver y Pickering, 1923). La regla de cálculo descrita por Weaver y Pickering (1923), fue útil para obtener, rápidamente la solución de algunos problemas que con frecuencia ocurrían en la navegación de dirigibles. A continuación, se formulan y resuelven tres de la serie de problemas que fueron originalmente propuestos. La solución de los problemas, utilizando las ecuaciones formuladas en las secciones anteriores, se compara con la solución obtenida al hacer uso de la regla. En la solución de los problemas se supone que el gas del dirigible es hidrógeno y que la atmósfera es estándar. En casi todos los problemas, se supone (al igual que lo hicieron Weaver y Pickering) que el dirigible tiene un volumen total de $6880.9 \mathrm{~m}^{3}$.

\section{Problema 1}

Se pide calcular la fuerza de flotación $F_{B}$ que tiene un dirigible de $6880.9 \mathrm{~m}^{3}$ cuando se encuentra a una altura $z=1524 \mathrm{~m}$, si el barómetro y el termómetro miden sobre la superficie terrestre $(z=0 \mathrm{~m}) 101305.5 \mathrm{~Pa}$ y 288.7 $\mathrm{K}$ respectivamente. Se supone que el hidrógeno tiene $95 \%$ de pureza.

Solución: Sobre la superficie terrestre, $\mathrm{z}_{1}=0 \mathrm{~m}, \mathrm{P}_{1}=101305.5 \mathrm{~Pa}$ y $\mathrm{T}_{1}=288.7 \mathrm{~K}$. Los pesos específicos del aire y del gas en la posición de referencia $z_{1}$ se calculan a partir de las siguientes ecuaciones:

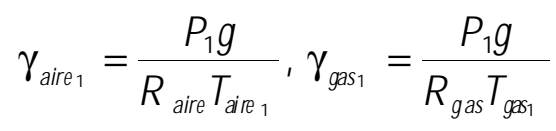

La constante del aire es $R_{\text {aire }}=287.04 \mathrm{~J} / \mathrm{kg}-\mathrm{K}$. La constante del gas se calcula a partir de la ecuación (10), $R_{\text {gas }}=R / M_{\text {gas }}=4124.26 \mathrm{~J} / \mathrm{kg}-\mathrm{K}$. Por tanto $\gamma_{\text {airg }}=11.98 \mathrm{~N} / \mathrm{m}^{3}$ y $\gamma_{9 \alpha_{1}}=0.834 \mathrm{~N} / \mathrm{m}^{3}$.

La fuerza de flotación $F B_{2}$, en la posición $z_{2}$, se obtiene a partir de la ecuación (21) (estado "taut") o partir de la ecuación (34) (estado "limp"), es decir:

$F_{B_{2}}=\mathrm{Vol}\left[\gamma_{\text {airg }}-\gamma_{\text {gag }}\right]=\mathrm{Vol}_{\text {gas } 2}\left[\gamma_{\text {airg }}-\gamma_{\text {gas }}\right]$ (40)

Debido a que el volumen del gas, en la posición $z_{2}$ se conoce, lo único que es necesario determinar, son los pesos específicos del aire y del gas, $\gamma_{\text {aire }}$ y $\gamma_{\text {䟨 }}$, respectivamente. Utilizando las ecuaciones 


$$
\frac{\gamma_{\text {aire }}}{\gamma_{\text {aire }}}=(1-a z)^{\frac{1}{n-1}}
$$

y

$$
\frac{\gamma_{\text {gas }}}{\gamma_{\text {gas } 1}}=(1-a z)^{\frac{n}{(k-1)}}
$$

en la ecuación (40), se obtiene:

$$
\begin{aligned}
& F_{B_{2}}=\mathrm{Vo} \mid\left[\gamma_{\text {arre }_{1}}(1-a \Delta z)^{\frac{1}{n-1}}-\gamma_{\text {gas }_{1}}(1-a \Delta z)^{\frac{n}{k(n-1)}}\right](43 \\
& \text { ) }
\end{aligned}
$$

donde: $\mathrm{V} \mathrm{ol}=6880.9 \mathrm{~m}^{3}, \Delta \mathrm{z}=1524 \mathrm{~m}, \mathrm{n}=1.234 \mathrm{y}$ $k=1.4$ (coeficiente politrópico del hidrógeno). El valor del parámetro a se calcula a partir de la ecuación (25), tomando en cuenta que $\mathrm{T}_{\text {air a }}=288.7 \mathrm{~K}$, por tanto, $\mathrm{a}=2.244048 \times 10^{-5}$ $\mathrm{m}^{-1}$. Sustituyendo los valores anteriores en la ecuación (43), se obtiene que $F_{B_{2}}=66008.91$ N. Weaver y Pickering (1923) sugieren alterar la fuerza de flotación por 0.95 (porcentaje de pureza del hidrógeno), por tanto, $F_{B_{2}}=$ $62708.46 \mathrm{~N}$. Weaver y Pickering reportan un valor de $F_{B_{2}}=62453 \mathrm{~N}$.

\section{Problema 2}

Se pide encontrar qué tan lleno debe estar un dirigible que se encuentra sobre la superficie terrestre, para que al elevarse hasta una altura de $2438.4 \mathrm{~m}$, no tenga que liberarse gas.

Solución: El dirigible se encuentra en la condición flex i ble. Si no se libera gas, $Q_{\text {gas }}=$ $\gamma_{\text {gas }_{1}} \vee \mathrm{Ol}_{\text {gas }_{1}}=\gamma_{\mathrm{gas}_{2}} \mathrm{Vol}_{\mathrm{gas}_{2}},($ Ecuación 26), por lo que puede escribirse

$$
\frac{\mathrm{V} \mathrm{ol}_{\text {gas }}}{\mathrm{V} \mathrm{ol}_{\text {gas }_{2}}}=\frac{\gamma_{\text {gas }_{2}}}{\gamma_{\text {gas }_{1}}}
$$

Utilizando la ecuación (42) en la expresión (44), se tiene

$$
\frac{\mathrm{Vol}_{\mathrm{gas}_{1}}}{\mathrm{Vol}_{\mathrm{gas}_{2}}}=(1-\mathrm{a} \Delta \mathrm{z})^{\frac{\mathrm{n}}{\mathrm{k(n-1)}}}
$$

en donde $\Delta z=2438.4 \mathrm{~m}$. Si en la ecuación (45) se utiliza el mismo valor de a evaluado en el ejemplo anterior, en donde se supone que $\mathrm{T}_{\text {aire }}=288.7 \mathrm{~K}$, el resultado es el siguiente

$$
\frac{\mathrm{V} \mathrm{Ol}_{\text {gas }_{1}}}{\mathrm{~V} \mathrm{O}_{\text {gas } 2}}=0.808
$$

El volumen inicial del di ri gi ble es el $80 \%$ del volumen que tendrá la nave a los $2438.9 \mathrm{~m}$ de altura (sin perder gas). Weaver y Pickering reportan $78 \%$.

\section{Problema 3}

La fuerza de flotación de un dirigible que se encuentra en equilibrio es $60050.9 \mathrm{~N}$. En estas condiciones la temperatura del aire es 272.03 K y la temperatura del gas es $280.37 \mathrm{~K}$. Si la nave no pierde gas, hasta el momento en que las temperaturas de los dos fluidos son iguales. ¿Cuál será la fuerza de flotación en ese momento?

Solución: El dirigible se encuentra en estado flex i ble. La fuerza de flotación $F_{B_{1}}$ puede escribirse en términos de la temperatura de los fluidos, es decir, (Ecuación 31) y la ecuación de estado (Ecuación1)

$$
F_{B_{1}}=Q_{\text {gas }}\left(\frac{\gamma_{\text {are }_{1}}}{\gamma_{\text {gas }_{1}}}-1\right)=Q_{\text {gas }}\left[\frac{\mathrm{R}_{\text {gas }_{\text {aire }}}}{\mathrm{R}_{\text {gas }_{1}}}-1\right]
$$


Si se conocen los valores de $F_{B_{1}}=60050.9 \mathrm{~N}$, $T_{\text {air e1 }}=272.03 \mathrm{~K}, T_{\text {gas } 1}=280.37 \mathrm{~K}, 1 / \sigma=R_{\text {gas }} / R_{\text {aire }}$ = 14.36. De la ecuación (47), puede obtenerse

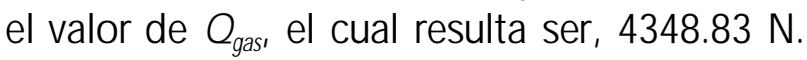
Dado que únicamente existe cambio de la temperatura del gas, puede utilizarse la expresión (37) en la forma siguiente

$$
\Delta F_{B_{1}}=Q_{\text {gas }}\left[\frac{\Delta \mathrm{T}_{\text {gas }}}{\sigma \mathrm{T}_{\text {aire }}}\right]
$$

donde $\Delta T_{\text {gas }_{1}}=272.03-280.37=-8.34 \mathrm{~K}$. Sustituyendo el valor de los parámetros en la ecuación (48), el resultado es $\Delta \mathrm{F}_{B_{1}}=-1915.65$ $N$. La fuerza de flotación $F_{B_{1}}$ se reduce entonces en $\Delta F_{B 1}$, dando como resultado 58135.24 N. Weaver y Pickering reportan $56803.79 \mathrm{~N}$.

\section{Conclusiones}

Con los nuevos materiales (como el titanio, kevlar, mylar, etc.), y con la capacidad actual que se tiene para predecir el comportamiento físico de los cuerpos sólidos deformables y de los fluidos (esfuerzos, deformaciones, estabilidad aerodinámica, predicción de características meteorológicas, etc.), es de esperarse que vuelva a surgir una nueva era de estos gigantes voladores. La teoría que se ha presentado es muy sencilla y fácil de comprender y el estudiante de ingeniería o física, interesado en el tema de los dirigibles, puede aprovecharla para incursionar en el diseño de estas naves.

\section{Referencias}

Arnstein K. y Klemperer W. (1936). Performance of Airships. In Aerodynamic
Theory, Vol. VI, Divi sion R, Volume VI, pp 49-64. (edited by W.F. Durand), BerlinJulius Springer.

Avila R. y Martínez E. (2001). Dirigibles flexibles (Blimps): Historia y aerostática. Boletín de la Sociedad M exicana de Física, 15(2):59-62.

Delahunt-Rimmer P. (1997). A Model to Evaluate the Commercial Applications of Airships. AIRSH IP, (118):26-30.

Hayes D.E. (1994). Airship Applications. AIRSH IP, (104):10-14.

Hillsdon R.H. (1992). An Anal ysis of Viable Roles for Airships in the 21st Century. AIRSH IP, (98):9-20.

Jacobo C. y Zimán D. (1984). El dirigible y el transporte público. Elementos, 2(12):19-24.

Kleinheins P. (1985). Die grossen Zeppeline: Die $G$ eschichte des Luftschiffbaus. VDI-Verlag.

Lana-Pliego F. (1991). Correspondence. AIRSH IP , (91):21-22.

Meyer P. (1980). Luftschiffe: Die Geschichte der Deutschen Zeppeline. Verlag Wehr \& Wissen.

Prandtl L. y Tietjens O.G. (1957). Fundamentals of Hydro \& A eromechanics. Dover Publications, Inc.

Reynolds W.C. y Perkins H.C. (1977). Engineering Thermodynamics. Mc. Graw-Hill, Second edition.

Rodríguez E. y Rodríguez E. (1987). Diagnóstico Económico-Tecnológico de los dirigibles como medio de transporte. Secretaría de Comunicaciones y Transportes, México, Dirección General de Desarrollo Tecnológico, elaborado por la Empresa Consultora "Estraegia, SC".

Sahlberg B. (1986). LTA Lighter than Air, Final Report 1985-A Feasibility Study. The Market, Technical And Infrastructural Prospects for LTA - Technology in 
Sweden. Technical Report STU-5531986, Styrelsen För Teknisk Utveckling, Swedish.

Taylor J.A. (1992). Airship Applicationsfor the First Decade of the Twenty-first Century. AIRSHIP, (98):20-31.

Weaver E.R. y Pickering S.F. (1923). An Airship Slide Rule. Technical Report 160, National Advisory Committee for Aero nautics, USA.

White W.J. (1976). Airships for the Future. Ster ling Publishing Co., Inc., New York.

\section{Semblanza del autor}

Rubén Á vila-Rodríguez. Realizó sus estudios de ingeniería mecánica en la Facultad de Ingeniería de la UNAM en donde obtuvo el doctorado con mención honorífica. Fue investigador del Instituto Nacional de Investigaciones Nucleares desde 1980 hasta 2001, año en que se incorporó a la Facultad de Ingeniería como profesor en el Departamento de Termoenergía y Mejoramiento Ambiental. Fue presidente de la División de Dinámica de Fluidos de la Sociedad Mexicana de Física. Actualmente es miembro del Sistema Nacional de Investigadores y o cupa la Cátedra Espe cial "Angel Borja O sorno", otorgada por la Facultad de Ingeniería. Ha publicado diversos trabajos originales de investigación y divulgación en revistas nacionales e internacionales, todos relacionados con las áreas de transferencia de calor, mecánica de fluidos, flujos turbulentos con partículas en suspensión y fenómenos con cambio de fase. Ha sido director y asesor de diversas tesis de doctorado, maestría y licenciatura; ha participado en numerosos congresos e impartido conferencias y cursos, dentro y fuera del país de temas en los que se especializa.

Jaime Cervantes de Gortari. Es ingeniero mecánico electricista (UNAM, 1970); maestro en ingeniería mecánica (UNAM, 1972); y doctor (Ph.D.) en ingeniería mecánica (Purdue Univer sity, 1976). Es autor y coautor de más de 120 publicaciones (libros de texto, apuntes, monografías de divulgación y artículos originales de investigación). Ha dictado numerosos cursos y conferencias en instituciones nacionales y extranjeras; ha sido asesor en estudios sobre investigación y educación en ingeniería, así como en proyectos relacionado s con el aprovechamiento energético en plantas industriales. Es miembro de honor de la Academia de Ingeniería y miembro regular de la Academia Mexicana de Ciencias. Actualmente es profesor de tiempo completo de la Facultad de Ingeniería de la UNAM y miembro del Sistema Nacional de Investigadores. En el año 2002, la UNAM le otorgó el Premio Universidad Nacional, Docencia en Ciencias Exactas. 